\title{
Characterization of a hand-wrist exoskeleton, READAPT, via kinematic analysis of redundant pointing tasks
}

\author{
Chad G. Rose ${ }^{\dagger}$, Fabrizio Sergi ${ }^{\dagger \S}$, Youngmok Yun ${ }^{\ddagger}$, Kaci Madden ${ }^{\ddagger}$, \\ Ashish D. Deshpande $\ddagger$, and Marcia K. O’Malley ${ }^{\dagger}$
}

\begin{abstract}
Training coordinated hand and wrist movement is invaluable during post-neurological injury due to the anatomical, biomechanical, and functional couplings of these joints. This paper presents a novel rehabilitation device for coordinated hand and wrist movement. As a first step towards validating the new device as a measurement tool, the device transparency was assessed through kinematic analysis of a redundant finger pointing task requiring synergistic movement of the wrist and finger joints. The preliminary results of this new methodology showed that wearing the robot affects the kinematic coupling of the wrist and finger for unconstrained pointing tasks. However, further experiments specifying a subset of the solution manifold did not exhibit the same difference between robot and no robot trials. The experiments and analysis form a promising method for the characterization of multi-articular wearable robots as measurement tools in robotic rehabilitation.
\end{abstract}

\section{INTRODUCTION}

Robotic rehabilitation has been successfully implemented for several neuromuscular conditions, providing a safe, accurate way to administer high intensity, long duration physical therapy, and record quantitative data about subjects' progress [1]. The rehabilitation of the hand and wrist is critical to restore the ability to perform activities of daily living (ADL).

Due to tendon and muscle anatomy, the wrist and hand are kinematically and dynamically linked [2]. The hand and wrist have biomechanical couplings as well, with previous studies identifying finger and wrist position-dependent passive properties of the hand [3]-[5]. Since most functional ADL involve coordinated movement of the hand and wrist, therapy for certain impairments, such as Parkinson's disease, focuses on regaining coordination [6]. Therefore, integrated hand and wrist therapy has the potential to improve the rehabilitative outcomes. Often, the anatomical, biomechanical, and functional couplings of the hand and wrist are overlooked in robotic rehabilitation. A number of hand and wrist exoskeletons have been separately developed [7][9], and the efficacy of robot-aided rehabilitation has been clinically verified [10]. Still, most devices do not allow for coordinated movement of the wrist and hand.

In addition to coordinated movement capabilities and functional workspace requirements [11] such as range of

This work was supported in part by grants NSF-CPS-1135949/1135916, and NSTRF- NNX13AM70H. ${ }^{\dagger}$ : Mechatronics and Haptic Interfaces Lab, Dept. of Mechanical Engineering, Rice University, Houston, TX 77005. $\S$ :Biomedical Engineering, University of Delaware, Newark, DE 19716. Corresponding author: cgr2@rice.edu. ${ }^{\ddagger}$ : ReNeu Robotics Lab, Dept. of Mechanical Engineering, University of Texas, Austin, TX 78712.

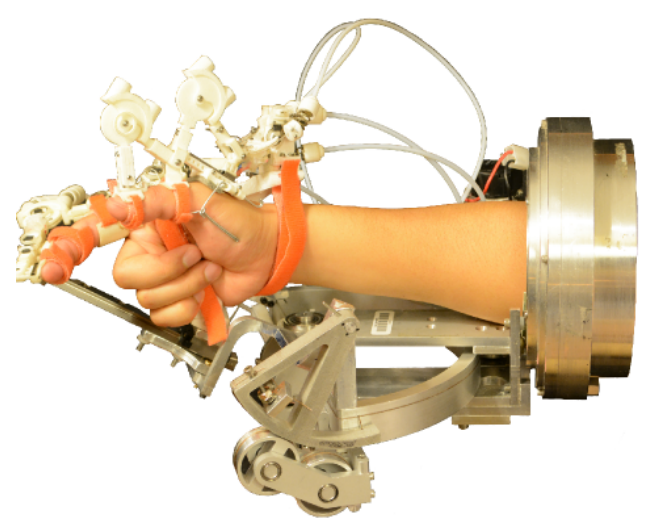

Fig. 1. The READAPT, shown with wrist, finger, and thumb DOF, is a novel integrated hand and wrist exoskeleton evaluated as a measurement device via kinematic analysis.

motion (ROM) and torque required for ADL, a rehabilitation robot must possess quantitative measurement capabilities for evaluation [12], requiring high quality position sensing, good backdrivability, and backlash-free operation [13]. Traditionally, quantitative measurement capabilities and backdrivability, also termed as device transparency, have been inferred from characterization metrics such as inertia, static friction, and viscous friction. While these traditional metrics are useful for comparisons of established devices [14], they are insufficient for validating them as measurement tools in synergistic multiarticular movements. Specifically, these metrics do not quantify the effect the devices have on the kinematic and kinetic movements properties. A direct comparison between movement measured by a robotic device and a 'ground truth' device is required to validate a device as a reliable measurement tool. This comparison should take the form of generalizable methods and metrics for motionbased device transparency assessments. Previous studies have used kinematic data to investigate wrist dynamics [15] and to characterize a wrist robot's effect on wrist pointing movements [16]. However, wrist pointing movements seem to be less 'smooth' and more variable than those of reaching movements, which complicates commonly used kinematic features for post-rehabilitation evaluation [17]. Despite this potential shortcoming, kinematic characteristics of coordinated movements are a prime target for investigations, since they can be measured in a non-invasive way, allowing for 
validation of an exoskeleton as a measurement device.

To address the need for coordinated wrist and hand therapy tool, this paper presents a novel hand and wrist exoskeleton in Section II. To validate this prototype as a measurement tool, Section III presents a motion-based transparency assessment of the device. The results in Section IV show the device's effect on hand and wrist kinematic couplings.

\section{READAPT: RoBOTIC EXOSKELETON TO ASSIST DistaL ÂRM PHYSICAL THERAPY}

The READAPT, shown in Fig. 1, leverages the design of existing devices [18], [19] to enable coordinated hand and wrist therapy. This device is designed to actuate eight degrees of freedom (DOF) of the hand (four at the thumb, two each for middle and index finger) and and three of the wrist, with passive constructions to prevent over-constraining the kinematics. As a therapy tool, the new device meets the functional workspace criteria for rehabilitation devices, shown in Table I. As a measurement device, the READAPT utilizes dynamic cancellation control for the hand and unpowered backdriving for the wrist DOF.

TABLE I

ADL REQUIREMENTS [20]-[22]AND READAPT CAPABILITIES [19]

\begin{tabular}{|c|cc|cc|}
\hline Joint & ROM & [deg] & Torque & {$[\mathbf{N m}]$} \\
& ADL & EXO & ADL & EXO \\
\hline Forearm pro./sup. & 150 & 180 & 0.06 & 1.69 \\
Wrist flex./ext. & 115 & 130 & 0.35 & 3.37 \\
Wrist radial/ulnar dev. & 70 & 75 & 0.35 & 2.11 \\
Index Finger MCP flex./ext. & 70 & 74 & 0.8 & 2.4 \\
\hline
\end{tabular}

The hand portion of the exoskeleton minimizes unnecessary forces on the finger by design and through control. In total, the hand portion of the exoskeleton has sixteen sensors to estimate all DOF of the three actuated fingers, with series-elastic-actuators (SEA) to control joint torques. These features also allow for estimation of the MCP, PIP, and distal interphalangeal (DIP) joint angles during flexion and extension, and the MCP joint angle during abduction and adduction. The mechanisms of the exoskeleton minimize any actuator-induced finger joint reaction forces and prevent misalignment between the robot joints and the human joints. The inertia of the hand-robot system is minimized to preserve the dexterity of hand movements and promote dynamic transparency of the device. Selective Laser Sintering (SLS), a type of additive manufacturing, of Nylon-12 plastic components and remote actuation via Bowden cables reduced the weight of the wearable portion of the device to a mere $75 \mathrm{~g}$. However, Bowden cables introduce a significant amount of friction, which prevents the exoskeleton from achieving passive backdriveability [23]. To overcome this friction, the exoskeleton utilizes active control methods to apply zero torque to the human joint. The applied force of the exoskeleton can be estimated by measuring the displacement of the SEA linear springs. Thus, the exoskeleton can achieve backdriveability if the displacement of the linear springs are controlled to be zero. Mechanical characteristics of the exoskeleton are listed in Table I. Note that the $70^{\circ}$ refers to $60^{\circ}$ of flexion and $10^{\circ}$ of extension and the $74^{\circ}$ refers to $72^{\circ}$ of flexion and $2^{\circ}$ of extension for ADL and device ROM, respectively.

The wrist portion of the device is a RRR mechanism with forearm pronation/supination, wrist flexion/extension and wrist radial/ulnar deviation DOF, actuated by DC motors and capstan cable transmissions, providing low friction and backlash-free operation. A thorough characterization of the wrist module was previously presented [24].

\section{EXPERIMENTAL METHODS}

We are interested in understanding the suitability of our exoskeleton as a rehabilitation assessment device. To this end, we devised a redundant planar pointing task using the index finger metacarpalphalangeal (MCP) and wrist flexion/extension joints. The selected task activates all joints of interest, enabling the identification of kinematic couplings and the analysis of their variation based on specific experimental conditions. The task is redundant, since the task solution space is defined by a 1D manifold of wrist and MCP joint angles, which can be categorized as either in-phase, where the MCP and wrist both flex or extend relative to an initial starting position, or out-of-phase, where the MCP and wrist joints rotate in opposite directions. In order to investigate different pose-dependent synergies, the pointing tasks are modeled after previous experiments [3] via obstacles to generate in-phase, and out-of-phase movements in addition to obstacle-free unconstrained tasks.

\section{A. Subjects}

Nine subjects, ages 21-30, eight female and one male, all right hand dominant and having no known neuromuscular deficit or hand injury, completed the study in compliance with the Rice University Institutional Review Board.

\section{B. Motion Capture}

Six Optitrack Flex V100R2 100 FPS cameras, $5 \mathrm{~mm}$ passive reflective markers, running in soft real time through Quarc and Simulink in Windows were used to measure the relative joint angles during the pointing tasks. Passive markers were placed on the distal phalange and the lateral

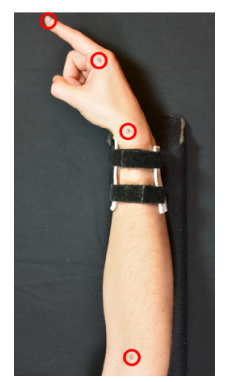

(a) NR Condition

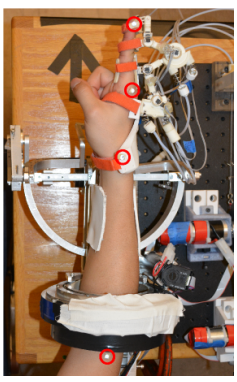

(b) $\mathrm{R}$ Condition

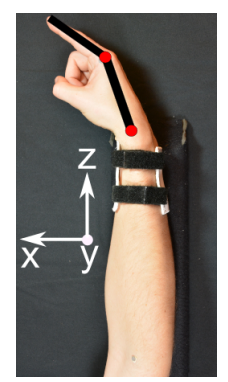

(c) Task Overlay
Fig. 2. Passive optical marker placement circled in red and their relationship to visualization, enabling robot and no robot kinematic comparisons 
elbow crease as line endpoints, and on the bony landmark for the MCP in a similar manner to related experiments [25], [26] and the top of the radial head [27] as joint axes, as seen in Fig. 2. As shown in Fig 2b, the radial head bony landmark was not readily visible for marker placement when wearing the device, so a secondary marker location above the radial head was selected, and was visually inspected to minimize travel during wrist flexion/extension. In order to prevent proximal interphalangeal (PIP) joint or distal interphalangeal (DIP) joint flexion/extension, subjects' fingers were taped and splinted. The 3D positions of each marker were projected onto a plane perpendicular to the rotation axes.

The joint angles, $\theta_{n}, n=1,2$, were determined by first approximating the four markers, numbered from proximal to distal, as coplanar endpoints of three lines $L_{i}, i=1,2,3$, then determining the relative angle between the lines:

$$
\theta_{n}=\operatorname{sign}\left(\left(L_{n} \times L_{n+1}\right) \cdot \hat{j}\right) \operatorname{acos}\left(\frac{L_{n} \cdot L_{n+1}}{\left\|L_{n}\right\|\left\|L_{n+1}\right\|}\right)
$$

The positive direction for each joint angle was defined to be counter-clockwise as viewed from above, so that for each joint, flexion was positive, and extension was negative, and would follow the right-handed rule convention for the world frame $y$ axis, shown in Fig. 2c and used in equation 1.

\section{Experimental Protocol}

The pointing tasks were conducted with three withinsubject factors: condition (robot (R) or no-robot (NR)), category of movement (unconstrained, in-phase, or out-of-phase), and target location (requiring wrist flexion or extension). Subjects completed the tasks first when using the READAPT as a measurement device, and then repeated the protocol without the robot. The pointing task was represented in a virtual display seen in Fig. 3 which maps the horizontal plane of the table onto the screen, imitating the view of looking down at your hand as you point, shown in Fig. 2c. The visualization consisted of two black lines, representing the hand and finger, and red circles representing joint axes, along with targets and obstacles. Subjects' forearms were constrained to a planar surface by the exoskeleton or a splint. Before the start of each task, subjects first had to match a specified pose of $0 \pm 3^{\circ}$ wrist flexion, and $20 \pm 3^{\circ}$ MCP flexion, estimated to be a comfortable, near-neutral position, shown in Fig. 3a as a thick gray outline of the starting pose. As soon as this start pose had been reached, subjects were shown a target (Fig. 3b), requiring either wrist flexion or extension, and one of three possible constraint

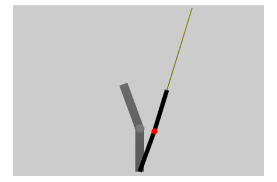

(a) Task start

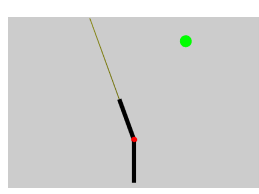

(b) Mid-task

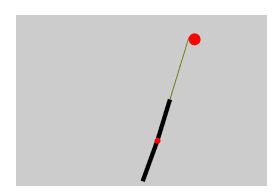

(c) Task completed
Fig. 3. Subjects match a starting pose, shown in gray, before completing the pointing task, in this case an unconstrained extension task conditions (unconstrained, in-phase, out-of-phase), shown in Fig. 4. Subjects were instructed to point quickly at each target as they appeared, and one second after reaching the target (Fig. 3c), the visualization disappeared, and subjects were instructed to return to a relaxed pose pointing along the $z$ axis in the world frame, for marker identification. Unconstrained targets had no obstacles (Fig. 4a, 4b), whereas in-phase (Fig. 4c, 4d) and out-of-phase (Fig. 4e, 4f) targets had obstacles requiring poses within a reduced solution manifold. Subjects performed a set of ten practice tasks, then fifty randomized tasks for each constraint condition (unconstrained, in-phase, out-of-phase).

\section{RESUlts AND ANALYsis}

A set of measures were defined to assess whether the interaction with the robotic device would change the handwrist coordination patterns observed during the redundant pointing tasks. Initial analysis has focused on the inter-peak time delay between wrist and finger joint velocity maxima, and the maximum straight line deviation of trajectories in a joint angle phase portrait. Both of these metrics examine coordination across the MCP and wrist flexion/extension joints, in that a coordinated movement is expected to consist of simultaneous multi-DOF movements with similar peak velocity times. Dis-coordination caused by the exoskeleton is hypothesized to distort synergistic multi-DOF trajectories into successions of single-DOF movements, which will increase straight line deviation in the joint angle phase portrait and the time between peaks in the joint velocity profiles.

\section{A. Data processing}

Individual tasks were segmented by assigning the start of the movement to the minimum of the absolute value of the sum of the MCP and wrist joint velocities after the start position had been reached (Fig. 3a), and the end of the movement to be one second after reaching the target (Fig. 3c). Segmenting in this manner provided more consistent results than using velocity thresholds due to the experimental design allowing subjects to 'swing-through' the initial or target position. Filtering and differentiation was accomplished via a third order Savitzky-Golay filter with a 21-sample (200 ms) window. For each combination of the three factors $a$ ) condition $b$ ) category of movement and $c$ ) target location, the inter-peak delay and straight line deviation metrics were averaged across subjects and analyzed through a factorial repeated measures ANOVA.

\section{B. Inter-peak time}

The first characteristic investigated is the time delay between the velocity peaks of the flexion-extension axes of the MCP and wrist, defined as:

$$
\Delta T=t_{p, w}-t_{p, M C P}
$$

Positive values of $\Delta T$ imply that the MCP velocity peak anticipates the wrist's. Fig. 5 shows the inter-peak times for all subjects, with each task grouped next to its corresponding 


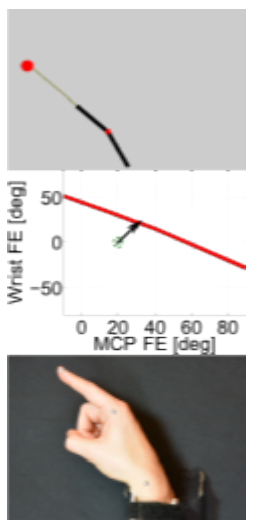

(a) Unconstr. flex.
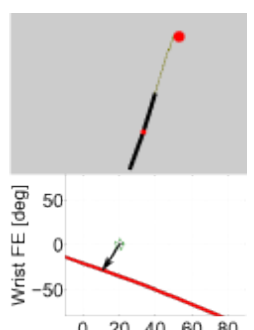

020
${ }^{2}$
MCP FE [deg]

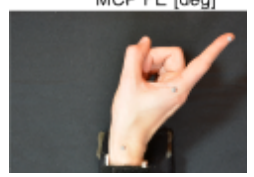

(b) Unconstr.ext.

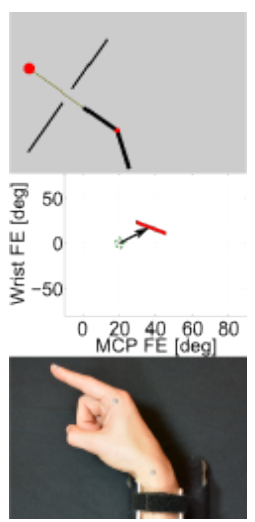

(c) In-Phase flex.
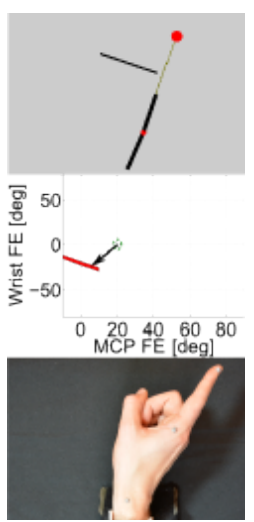

(d) In-Phase ext.
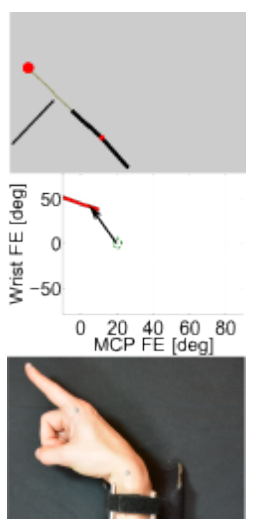

(e) Out-of-Phase flex.
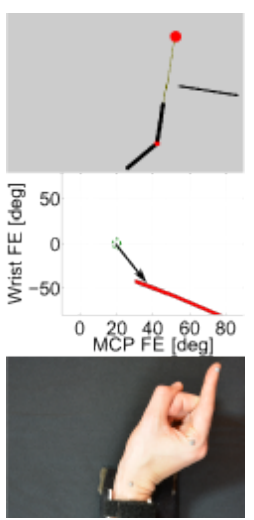

(f) Out-of-Phase ext.

Fig. 4. Each subfigure shows one of the six experimental tasks, with the visualization presented to the subject on top, mapped as shown in Fig. 2c. The middle figure is the solution manifold, shown in red of joint angles that successfully reach the target and avoid the obstacle, plotted over the workspace. MCP and wrist flexion/extension ROM are the horizontal and vertical axes, respectively. The bottom figure shows the pose required to complete the task.

$\mathrm{R} / \mathrm{NR}$ condition. Inter-peak time means were considered outliers if they fell outside of three interquartile ranges from the $25^{\text {th }}$ or $75^{\text {th }}$ percentile. The ANOVA showed an interaction between robot/no-robot and category of movement $\left(F(1.26,10.05)=5.4, p=0.04, \eta_{p}^{2}=.40\right)$. This interaction was decomposed using simple main effects, summarized in Table II, which shows an effect for unconstrained and inphase movements, but not for out-of-phase movements.

The tight grouping of unconstrained and in-phase no-robot inter-peak times around zero, suggests that natural pointing tasks involve simultaneous finger and wrist movement, as shown in blue in the first four columns of Fig. 5. The robot condition perturbed this coupling by increasing the magnitude and variability of inter-peak time, which could be attributed to the robot's inertial and friction characteristics. However, this statistically significant difference was not found in the out-of-phase movements, which may be unfamiliar, and do not possess the same strong coordination as familiar in-phase movements.

TABLE II

EFFECT OF ROBOT ON INTER-PEAK TIME

\begin{tabular}{|c|ccc|}
\hline SME & $\mathrm{F}(1,8)$ & $\mathrm{p}$ & $\eta_{p}^{2}$ \\
\hline Unconstrained & 28.6 & 0.001 & 0.781 \\
In-Phase & 36.7 & $<0.001$ & 0.82 \\
Out-of-phase & 3.6 & 0.095 & 0.31 \\
\hline
\end{tabular}

\section{Straight line deviation}

Analysis of the trajectories in the phase plane defined by joint angles enables the assessment of linearity of synergistic hand and wrist rotations. Fig. 6 shows representative trajectories in both $\mathrm{R}$ and NR conditions. The decrease in coordinated movement results in a greater curvature of the trajectory in the phase plane for the $\mathrm{R}$ condition, which was characterized by the maximum deviation from a straight line path for each trajectory. The first and final positions of

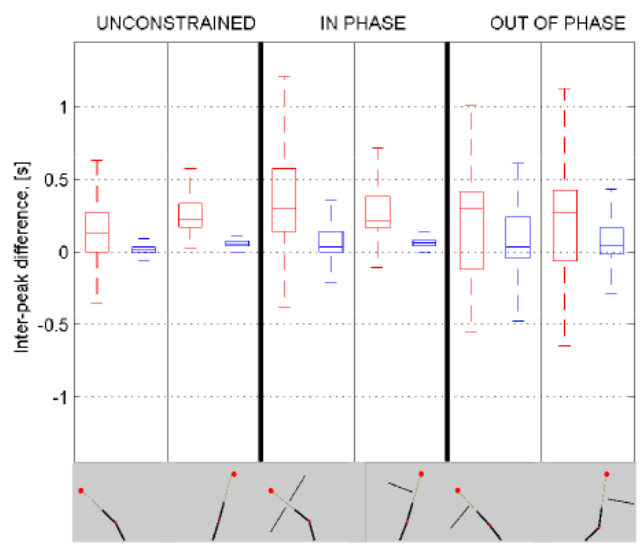

Fig. 5. Inter-peak time delay between finger and wrist velocities shows significant difference in unconstrained and in-phase conditions between robot (left in red in each sub plot) and no-robot (right in blue) conditions.

the filtered and segmented position trajectories were used to create a straight line path, and straight line deviation is defined as the maximum distance between the measured profile and the calculated straight line path. Mean deviations for all subjects are shown in Fig. 7. The ANOVA showed an interaction between robot/no-robot and category of movement $\left(F(1.45,11.6)=17.35, p=0.001, \eta_{p}^{2}=.68\right)$. This

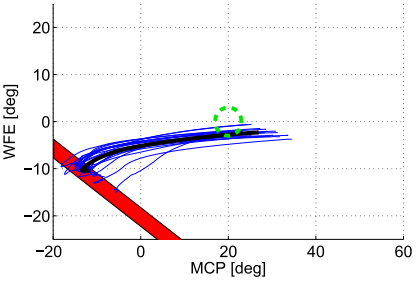

(a) NR Unconstrained Extension

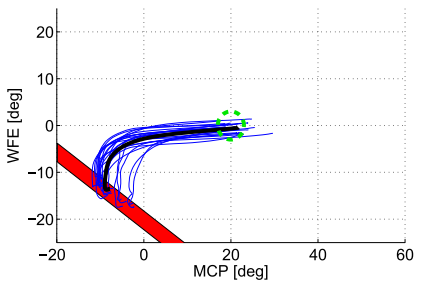

(b) R Unconstrained Extension
Fig. 6. Comparison of robot (R) and no robot (NR) tasks, with the solution manifold in red, starting position represented by a green circle, individual paths in blue, and an average path in thick black. 


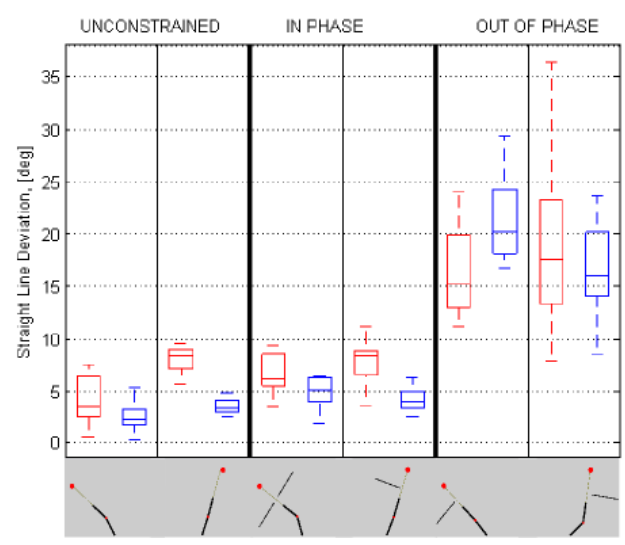

Fig. 7. Maximum straight line deviation for all subjects shows difference in unconstrained and in-phase conditions between robot (left in red in each sub plot) and no-robot (right in blue in each sub plot) conditions.

interaction was decomposed using simple main effects, which showed an effect for unconstrained and in-phase movements, but not for out-of-phase movements, and the results are summarized in Table III.

TABLE III

EFFECT OF ROBOT ON STRAIGHT LINE DEVIATION

\begin{tabular}{|c|ccc|}
\hline SME & $\mathrm{F}(1,8)$ & $\mathrm{p}$ & $\eta_{p}^{2}$ \\
\hline Unconstrained & 20.4 & 0.002 & 0.710 \\
In-Phase & 26.6 & 0.001 & 0.769 \\
Out-of-phase & 2.1 & 0.185 & 0.208 \\
\hline
\end{tabular}

\section{Discussion}

As expected, the robot affected the kinematic coupling of the hand and wrist as measured by inter-peak time delay and maximum straight line deviation. The robot possesses non-negligible inertial and friction characteristics that should alter movement. While models of wrist dynamics [15] show limited inertial effects on pointing movement dynamics, the inertia of the READAPT, approximately the same as the wrist module, [24] is nearly a five-fold increase over the inertia used in a biomechanical model, [15] which could have an effect on wrist pointing movements. Since static friction is not present in the model, it could have an even greater perturbing effect on kinematic couplings. Second, there is a distinction on the types of movement, as neither metric showed a significant difference between robot and no robot conditions for out-of-phase movements. It is possible that this dependency on phase is the result of familiarity with inphase movements, which are more commonplace than outof-phase movements. Commonplace movements may have more refined, and potentially sensitive, internal models [28], whereas the out-of-phase models are less developed and potentially less likely to be disturbed.

The difference in straight line deviation between robot and no-robot conditions can be explained by two observations. First, most subjects avoided or delayed moving the wrist during robot condition experiments as compared to the norobot condition experiments. This delay was likely due to the inertia and static friction of the READAPT at the wrist, which caused the discoordination. These results suggest a need to reduce the inertia of the device through the implementation of lightweight materials and further material reduction when possible. The frictional characteristics of the device need to be reduced through feedforward cancellation, to further improve the transparency of the device. Second, MCP flexion/extension range of motion seemed to be limited for subjects by varying degrees, with an example shown in Fig. 6b. This variable decrease in ROM may have been caused by limitations of the particular interface components of the hand module of the exoskeleton. Inclusion criteria for this study centered on finger length, but individuals with similar finger lengths do not necessarily have the same diameter fingers, and the sub-optimal fit of the interfacial parts for the exoskeleton prevented full range of motion. These plots show that although subjects were able to fit inside the exoskeleton, they are still sensitive to improper interfacing, supporting the need for further optimization or customizable interface components.

During certain out-of-phase, flexion, no robot tasks (Fig. 4e), another kinematic coupling was observed, circled in Fig. 8. It appears that the wrist drags the finger into flexion before the finger extends to complete the task. This behavior was exhibited too frequently to be readily attributed to user error, as it did not appear consistently in all trials or in all subjects. The presence of the coupling did not seem to change over with training. It is hypothesized that the difficulty of the out-of-phase tasks could have contributed to its sporadic appearance, and further experiments aimed specifically at this new coupling are warranted.

These results indicate a need for further analysis of the device's effects on pointing tasks, both with regard to a deeper analysis of results, and recommendations for modifications to our experimental protocol. In future analysis, we will explore inter-peak delay over tasks, as well as its variance as a function of impedance. It is hypothesized that after training this delay could be compensated for by healthy subjects for a constant impedance, and that at lower levels of impedance, this variance will be reduced. Therefore, comparisons between impedances of varying levels across the

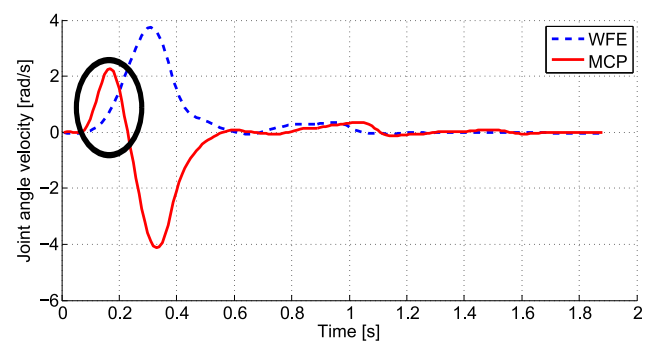

Fig. 8. Kinematic coupling (circled) appearing in select out of phase tasks. The finger anticipates wrist flexion before extending to accomplish the task. 
different joints of the exoskeleton are planned.

Regarding our experimental methods, subjects were allowed to complete a task as soon as they reached the requisite pose, causing difficulties in segmenting the movements, requiring the changed segmentation procedure which still resulted in some skewed start positions in Fig. 6. All future experiments should require that subjects reach and remain on the target for a prescribed amount of time, or have some velocity threshold that they must remain below before being allowed to proceed, to prevent the 'swing-through' completions of tasks. To increase measured joint angle accuracy, experiments should consider performing initial calibrations [29], [30] to determine MCP and wrist flexion/extension axes. This calibration process will also avoid the difficulty of locating joint axes with a passive marker, and prevent the visual inspection required in Section III-B. Finally, having more interfacing components in a wider variety of sizes could prevent some of the ROM limitations identified in Section IV$\mathrm{C}$ and widen the inclusion criteria.

\section{CONCLUSION}

The READAPT is a step towards an integrated hand and wrist exoskeleton to train coordinated wrist and hand movements following a neurological injury. The assessment method presented in this paper is a first step in quantifying the relationship between device transparency and the kinematic coupling of the hand and wrist during synergistic movements. The preliminary results show that hand and wrist kinematic couplings are sensitive to the inertial and friction disturbances and kinematic constraints of exoskeletons.

\section{ACKNOWLEDGMENTS}

The authors acknowledge Utku Pehlivan for the wrist module design, and Priyanshu Agarwal and Jonas Fox for their effort in designing the index finger module.

\section{REFERENCES}

[1] A. A. Blank, J. A. French, A. U. Pehlivan, and M. K. OMalley, "Current trends in robot-assisted upper-limb stroke rehabilitation: promoting patient engagement in therapy," Current Physical Medicine and Rehabilitation Reports, vol. 2, no. 3, pp. 184-195, 2014.

[2] Z.-M. Li, "The influence of wrist position on individual finger forces during forceful grip," The J. of Hand Surgery, vol. 27, no. 5, pp. 886896, 2002.

[3] A. D. Deshpande, N. Gialias, and Y. Matsuoka, "Contributions of intrinsic visco-elastic torques during planar index finger and wrist movements," Biomedical Engineering, IEEE Trans. on, vol. 59, no. 2, pp. 586-594, 2012.

[4] P.-H. Kuo and A. D. Deshpande, "Contribution of passive properties of muscle-tendon units to the metacarpophalangeal joint torque of the index finger," in Biomedical Robotics and Biomechatronics (BioRob), IEEE RAS and EMBS Intl. Conf. on, 2010, pp. 288-294.

[5] J. S. Knutson, K. L. Kilgore, J. M. Mansour, and P. E. Crago, "Intrinsic and extrinsic contributions to the passive moment at the metacarpophalangeal joint," J. of Biomechanics, vol. 33, no. 12, pp. 1675-1681, 2000.

[6] N. Dounskaia, A. W. Van Gemmert, B. C. Leis, and G. E. Stelmach, "Biased wrist and finger coordination in Parkinsonian patients during performance of graphical tasks," Neuropsychologia, vol. 47, no. 12, pp. 2504-2514, 2009.
[7] C. N. Schabowsky et al., "Development and pilot testing of HEXORR: Hand EXOskeleton Rehabilitation Robot," J. of Neuroengineering and Rehabilitation, vol. 7, no. 36, pp. 1-16, 2010.

[8] M. Bouzit, G. Burdea, G. Popescu, and R. Boian, "The Rutgers Master II-new design force-feedback glove," Mechatronics, IEEE/ASME Trans. on, vol. 7, no. 2, pp. 256-263, 2002.

[9] M. Cempini, M. Cortese, and N. Vitiello, "A Powered Finger-Thumb Wearable Hand Exoskeleton With Self-Aligning Joint Axes," Mechatronics, IEEE/ASME Trans. on, vol. 20, no. 2, pp. 705-716, 2015.

[10] P. S. Lum, S. B. Godfrey, E. B. Brokaw, R. J. Holley, and D. Nichols, "Robotic approaches for rehabilitation of hand function after stroke," American J. of Physical Medicine \& Rehabilitation, vol. 91, no. 11, pp. S242-S254, 2012.

[11] A. Schiele and F. C. van der Helm, "Kinematic design to improve ergonomics in human machine interaction," IEEE TNSRE, vol. 14, no. 4, pp. 456-469, 2006.

[12] O. Celik, M. K. O'Malley, C. Boake, H. S. Levin, N. Yozbatiran, and T. A. Reistetter, "Normalized movement quality measures for therapeutic robots strongly correlate with clinical motor impairment measures," IEEE TNSRE, vol. 18, no. 4, pp. 433-444, 2010.

[13] V. Hayward and K. E. MacLean, "Do it yourself haptics: part I," Robotics \& Automation Mag., IEEE, vol. 14, no. 4, pp. 88-104, 2007.

[14] J. A. French, C. G. Rose, and M. K. OMalley, "System characterization of MAHI Exo-II: A robotic exoskeleton for upper extremity rehabilitation," in ASME Dynamic Systems and Control Conf., 2014.

[15] S. K. Charles and N. Hogan, "Dynamics of wrist rotations," J. of Biomechanics, vol. 44, no. 4, pp. 614-621, 2011.

[16] N. L. Tagliamonte, M. Scorcia, D. Formica, D. Campolo, and E. Guglielmelli, "Effects of impedance reduction of a robot for wrist rehabilitation on human motor strategies in healthy subjects during pointing tasks," Advanced Robotics, vol. 25, no. 5, pp. 537-562, 2011.

[17] L. H. Salmond and S. K. Charles, "Why are wrist rotations considerably less smooth than reaching movements?" Emerging Ideas in Biomedical Research, 2012.

[18] P. Agarwal, Y. Yun, K. Madden, J. Fox, and A. D. Deshpande, "Exoskeleton for hand-wrist rehabilitation," http://www.me.utexas.edu/ $\sim$ reneu/res/handexo.html, Accessed 2015-03-25.

[19] A. U. Pehlivan, F. Sergi, A. Erwin, N. Yozbatiran, G. Francisco, and M. K. O'Malley, "Design and validation of the RiceWrist-S exoskeleton for robotic rehabilitation after incomplete spinal cord injury," Robotica, vol. 32, pp. 1415-1431, 2014.

[20] H. Hayashi and H. Shimizu, "Essential motion of metacarpophalangeal joints during activities of daily living," Journal of Hand Therapy, vol. 26, no. 1, pp. $69-74,2013$.

[21] T. Milner and D. Franklin, "Characterization of multijoint finger stiffness: dependence on finger posture and force direction," Biomedical Eng., IEEE Trans. on, vol. 45, no. 11, pp. 1363-1375, Nov 1998.

[22] J. C. Perry, J. Rosen, and S. Burns, "Upper-limb powered exoskeleton design," Mechatronics, IEEE/ASME Trans. on, vol. 12, no. 4, pp. 408417, Aug. 2007.

[23] D. Chen, Y. Yun, and A. Deshpande, "Experimental characterization of bowden cable friction," in IEEE International Conference on Robotics and Automation, 2014.

[24] A. U. Pehlivan, F. Sergi, and M. K. O'Malley, "A subject-adaptive controller for wrist robotic rehabilitation," Mechatronics, IEEE/ASME Trans. on, vol. 20, no. 3, pp. 1338-1350, June 2015.

[25] N. A. Baker, R. Cham, E. H. Cidboy, J. Cook, and M. S. Redfern, "Kinematics of the fingers and hands during computer keyboard use," Clinical Biomechanics, vol. 22, no. 1, pp. 34 - 43, 2007.

[26] P. Braido and X. Zhang, "Quantitative analysis of finger motion coordination in hand manipulative and gestic acts," Human Movement Science, vol. 22, no. 6, pp. $661-678,2004$.

[27] R. Schmidt, C. Disselhorst-Klug, J. Silny, and G. Rau, "A markerbased measurement procedure for unconstrained wrist and elbow motions," J. of Biomechanics, vol. 32, no. 6, pp. 615-621, 1999.

[28] D. M. Wolpert, Z. Ghahramani, and M. I. Jordan, "An internal model for sensorimotor integration," Science-AAAS-Weekly Paper Edition, vol. 269, no. 5232, pp. 1880-1882, 1995.

[29] Y. Yun, P. Agarwal, and A. D. Deshpande, "Accurate, robust, and realtime pose estimation of finger," J. of Dynamic Systems, Measurement, and Control, vol. 137, no. 3, p. 034502, 2015.

[30] E. Biryukova, A. Roby-Brami, A. Frolov, and M. Mokhtari, "Kinematics of human arm reconstructed from spatial tracking system recordings," J. of Biomechanics, vol. 33, no. 8, pp. 985 - 995, 2000. 